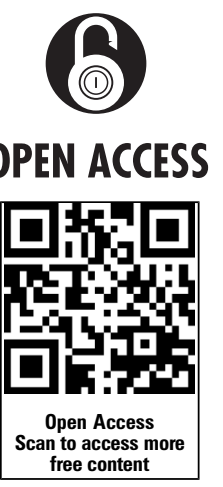

Handling editor Tore $\mathrm{K}$ Kvien

- Additional material is published online only. To view please visit the journal online (http://dx.doi.org/10.1136/ annrheumdis-2015-207490)

${ }^{1}$ Nuffield Department of Orthopaedics, Rheumatology and Musculoskeletal Sciences, University of Oxford, Oxford UK

${ }^{2}$ National Institute for Health Research Oxford

Musculoskeletal Biomedical Research Unit, Oxford, UK ${ }^{3}$ National Institute for Health Research Oxford

Comprehensive Biomedical Research Centre, Botnar Research Centre, Nuffield Orthopaedic Centre, Oxford, UK

${ }^{4}$ Division of Clinical Neurology, Nuffield Department of Clinical Neurosciences, John Radcliffe Hospital, University of Oxford, Oxford, UK

${ }^{5}$ Wellcome Trust Centre for Human Genetics, University of Oxford, Oxford, UK

\section{Correspondence to}

Professor B Paul Wordsworth, NIHR Oxford Musculoskeletal Biomedical Research Unit, Botnar Research Centre, Nuffield Orthopaedic Centre, Windmill Road, Headington, Oxford OX3 7LD, UK; paul. wordsworth@ndorms.ox.ac.uk

Received 20 February 2015 Accepted 19 August 2015 Published Online First 9 October 2015

\section{CrossMark}

To cite: Vecellio $M$, Roberts AR, Cohen CJ, et al. Ann Rheum Dis

2016;75:1534-1540.

\title{
The genetic association of RUNX3 with ankylosing spondylitis can be explained by allele-specific effects on IRF4 recruitment that alter gene expression
}

\author{
Matteo Vecellio, 1,2,3 Amity R Roberts, ${ }^{1,2,3}$ Carla J Cohen, 1,2,3 Adrian Cortes, ${ }^{4,5}$ \\ Julian C Knight, ${ }^{5}$ Paul Bowness, ${ }^{1,2,3}$ B Paul Wordsworth ${ }^{1,2,3}$
}

ABSTRACT

Objectives To identify the functional basis for the genetic association of single nucleotide polymorphisms (SNP), upstream of the RUNX3 promoter, with ankylosing spondylitis (AS).

Methods We performed conditional analysis of genetic association data and used ENCODE data on chromatin remodelling and transcription factor (TF) binding sites to identify the primary AS-associated regulatory SNP in the RUNX3 region. The functional effects of this SNP were tested in luciferase reporter assays. Its effects on TF binding were investigated by electrophoretic mobility gel shift assays and chromatin immunoprecipitation. RUNX3 mRNA levels were compared in primary CD8+ T cells of AS risk and protective genotypes by real-time PCR.

Results The association of the RUNX3 SNP rs4648889 with AS $\left(p<7.6 \times 10^{-14}\right)$ was robust to conditioning on all other SNPs in this region. We identified a $2 \mathrm{~kb}$ putative regulatory element, upstream of RUNX3, containing rs4648889. In reporter gene constructs, the protective $r 54648889$ ' $G$ ' allele increased luciferase activity ninefold but significantly less activity (4.3-fold) was seen with the $A S$ risk ' $A$ ' allele $(p \leq 0.01)$. The binding of Jurkat or CD8+ T-cell nuclear extracts to the risk allele was decreased and IRF4 recruitment was reduced. The AS-risk allele also affected H3K4Me1 histone methylation and associated with an allelespecific reduction in RUNX3 mRNA ( $p<0.05)$.

Conclusion We identified a regulatory region upstream of RUNX3 that is modulated by rs4648889. The risk allele decreases TF binding (including IRF4) and reduces reporter activity and RUNX3 expression. These findings may have important implications for understanding the role of $T$ cells and other immune cells in AS.

\section{INTRODUCTION}

Ankylosing spondylitis (AS) is a common form of spondyloarthropathy (SpA) with numerous robust genetic associations, of which most are currently unexplained. Some functional protein-coding single nucleotide polymorphisms (SNPs) have been identified in genes associated with AS, such as $H L A-B$, ERAP1 and IL23R. ${ }^{1-4}$ More commonly, the associated SNPs lie in non-coding flanking sequences or intragenic regions where they may influence gene expression. ${ }^{1}{ }^{2} 5$ Genome-wide association studies (GWAS) show convincing association between RUNX3 and AS and psoriatic arthritis, another form of SpA. ${ }^{126}$ This association extends to a cluster of SNPs at the RUNX3 locus, ${ }^{1}$ located from 0.5 to $2 \mathrm{~kb}$ upstream of the RUNX3 promoter. One of these, rs 4648889, lies in a putative transcription regulatory region defined by DNaseI hypersensitivity and the presence of chromatin marks correlated with active enhancers (H3K4Me1 and H3K27Ac). ${ }^{7}$ RUNX3 is a member of the runt domain-containing family of transcription factors (TFs), which play key roles in many developmental pathways. ${ }^{8}$ The $R u n x 3^{-/-}$mouse shows a complex phenotype affecting several organs, highlighting the broad spectrum of RUNX3 action. ${ }^{9}$ RUNX3 influences several types of immune cell that could be involved in AS, including natural killer (NK) cells and regulatory $\mathrm{T}$ cells, ${ }^{10}{ }^{11}$ but seems particularly involved in the differentiation and development of CD8 + T cells. ${ }^{12} 13$ It seems relevant that $\mathrm{CD} 8+\mathrm{T}$-cell numbers are reduced in AS and that this is related to the RUNX3 genotype. ${ }^{12}$ There is evidence for epigenetic regulation of RUNX3 expression; the gene has two differentially expressed alternative promoters resulting in proteins that have dissimilar transcriptional activation capacity. ${ }^{1415}$ The distal promoter is specifically demethylated in T cells ${ }^{16}$ but is methylated on both alleles in clonal cell populations. ${ }^{17}$ RUNX3 promoter methylation status has also been incriminated in acute myeloid leukaemia ${ }^{18}$ and malignant transformation of ovarian endometriosis. ${ }^{19}$

Numerous SNPs in a $\sim 15 \mathrm{~kb}$ linkage disequilibrium (LD) block upstream of RUNX3 are strongly associated with AS. In the recent large ImmunoChip study, the lead SNP was rs6600247 $\left(\mathrm{p}=1.3 \times 10^{-14}\right)$, which is in complete LD with rs4648889 ( $2 \mathrm{~kb}$ upstream of the RUNX3 promoter). ${ }^{1}$ Here we apply conditional analysis to investigate the $\mathrm{LD}$ patterns within this region to identify the SNP(s) most likely to be primarily associated with AS. We also use historic ENCODE data on the transcriptional and epigenetic regulation of RUNX $3,{ }^{20}$ together with in vitro and ex vivo molecular assays to investigate functional effects of rs4648889 on RUNX3 expression.

\section{METHODS \\ Genotyping}

Historical typing data from the AS ImmunoChip study ${ }^{1}$ were available for some patients or were otherwise obtained using TaqMan SNP assay (Life Technologies, Paisley, UK) to assign rs4648889 genotypes. Where required, DNA was extracted using the Qiagen AllPrep DNA/RNA Mini Kit (Qiagen, Manchester, UK). 


\section{Imputation}

Genotype data from the UK cases in the International Genetics of Ankylosing Spondylitis Consortium AS ImmunoChip study and WTCCC2 controls $^{1}$ were used to localise primary association signals. Haplotypes were inferred using SHAPEIT $^{21}$ with default parameters. Untyped variants were imputed using haplotypes from phase III of the 1000 Genomes Project (October 2014 release) ${ }^{22}$ and using IMPUTE V.2. ${ }^{23}$ Association analysis with a Bayesian logistic model accounting for uncertainty in imputed variants (score method) was performed using SNPTEST. ${ }^{24}$ Population structure was accounted for by including 10 principal components (PCs) as covariates in the regression analysis. Evidence for association is reported as the Bayes factor comparing the model of association with no association. The default priors in SNPTEST for the analysis were used.

\section{Luciferase reporter assay}

The $250 \mathrm{bp}$ sequence flanking $r s 4648889$ was amplified from human genomic DNA and cloned into the TA cloning kit pCR2.1 vector (Invitrogen, Paisley, UK), then subcloned into the pGL4.23(luc2/minP) reporter vector (Promega, Madison, Wisconsin, USA) at the SacI/XhoI restriction sites (primer sequences available on request). Point mutations corresponding to genetic variants (G/A) of rs4648889 were introduced using the QuikChange II XL Site-Directed Mutagenesis Kit (Agilent, Santa Clara, California, USA). Luciferase reporter assay details are available online (see online supplementary methods section).

\section{Patients with AS}

Following informed consent (COREC 06/Q1606/139 and OXREC B 07/Q1605/35), venous blood samples were obtained from 19 patients with positive HLA-B27 (average age 51 years, range 29-72) of white British ancestry fulfilling the modified New York criteria for AS. ${ }^{25}$ Sixteen were taking non-steroidal anti-inflammatory analgesics, two were on sulfasalazine but none were currently taking corticosteroids or immunosuppressants. None of the cases had ever received biological therapy, but four with active disease were sampled immediately before starting biological therapy. Twelve cases had low disease activity $(<4 / 10)$ measured by the Bath AS Disease Activity Index (BASDAI) ${ }^{26}$ but overall there was a considerable range of disease activity (mean BASDAI 4.8/10, range 0.7-10) and $\mathrm{C}$ reactive protein (CRP) (mean $17 \mathrm{mg} / \mathrm{L}$, range $0.5-74$ ).

\section{CD8+ T-cell isolation}

CD8 + T cells were isolated from peripheral blood mononuclear cells using the CD8 + T-cell Isolation Kit (Miltenyi, Bisley, UK), according to manufacturer's instructions. CD8 + T cells were then plated for $4 \mathrm{~h}$ in RPMI supplemented with $10 \%$ fetal bovine serum before harvesting for experiments.

\section{Electrophoretic mobility gel shift assay}

Nuclear extract from Jurkat cells was prepared using the Thermo Scientific NE-PER Nuclear and Cytoplasmic Extraction kit (Thermo Scientific, Waltham, Massachusetts, USA). Electrophoretic mobility gel shift assays (EMSAs) were performed with LightShift Chemiluminescent EMSA Kit (Thermo Scientific, Waltham, Massachusetts, USA) using $5 \mu \mathrm{g}$ of nuclear extract and $0.6 \mathrm{ng}$ biotin-labelled double-stranded oligonucleotides (50 bp fragment-Eurofins, Wolverhampton, UK). The sequences of the synthetic single-stranded oligonucleotides used in the construction of these double-stranded oligonucleotides are listed in the online supplementary methods.
Probes were prepared using a biotin $3^{\prime}$ end DNA labelling kit (Thermo Scientific, Waltham, Massachusetts, USA).

Single-stranded biotinylated oligonucleotides were mixed and annealed at room temperature for $1 \mathrm{~h}$. Unlabelled competitor probes were used in 100-fold excess.

EMSAs were performed according to standard protocol (Thermo Scientific).

Band intensity was quantified with ImageJ software (Bethesda, Maryland, USA).

A detailed protocol is available online (see online supplementary methods section).

\section{Chromatin immunoprecipitation-PCR}

Chromatin immunoprecipitation (ChIP) was performed using the Diagenode Low Cell\# ChIP kit (Liege, Belgium). For each ChIP, $6 \times 10^{4} \mathrm{CD} 8+\mathrm{T}$ cells were incubated with $1 \%$ formaldehyde for $10 \mathrm{~min}$ and $1.25 \mathrm{M}$ glycine added for $5 \mathrm{~min}$. DNA isolation was performed with DNA isolation buffer supplied by the kit. Quantitative PCR (qPCR) was performed on immune complex-associated DNA using allele-specific primers for rs4648889 (common forward primer: 5'-CCCTACGTGCTTTG CTGTTT-3', AS risk 'A' allele reverse primer: 5'-GGGCCTGG ACTCAGGTGT-3', AS-protective ' $G$ ' allele reverse primer: 5'-GGGCCTGGACTCAGGTGC-3'), detected with SYBR Green on ABI ViiA7 PCR instruments (Applied Biosystems, Paisley, UK). A compensatory factor $((\log (100) / \log 2)$ was subtracted from the cycle threshold $(\mathrm{Ct})$ values of the diluted input $(1 \%)$ in order to calculate the $\mathrm{Ct}$ values of the $100 \%$ input. Calculation of relative enrichment was done as follows: signals obtained from the ChIP are divided by signals obtained from input sample (representing the amount of the chromatin used in the ChIP $)=2^{\wedge}($ adj input-ct(IP)).

Relative occupancy was calculated as a ratio of specific signal over background: $\%$ input (specific loci)/\% input (background loci).

The antibodies used were: IRF4 (Santa Cruz Biotechnology, Dallas, Texas, USA, sc-377383), H3K4Me1 (Diagenode, Liege, Belgium, C15200150) and IgG (Diagenode, Liege, Belgium, C15200001).

\section{Quantitative real-time PCR}

RNA was isolated with TRIzol (Invitrogen, Paisley, UK) and cDNA synthesis (for $500 \mathrm{ng}$ RNA) was prepared with Superscript III from Invitrogen (Paisley, UK). A final concentration of $5 \mathrm{ng} / \mu \mathrm{L}$ was used in $\mathrm{qPCR}$, which was performed with the ABI ViiA7 PCR instrument (Applied Biosystems, Paisley, UK) using SYBR Master mix (Applied Biosystems, Paisley, UK) with evaluation of dissociation curves. mRNA levels of each gene were quantified using the $\Delta \Delta \mathrm{Ct}$ method and normalised to $\beta$-actin. For each gene, PCR melting curves were checked to evaluate the single, specific product.

The specific primers designed were RUNX3 forward: $5^{\prime}$-ACT CAG CAC CAC AAG CCA CT-3' RUNX3 reverse: 5'-GTC GGA GAA TGG GTT CAG TT-3' RUNX3 values were normalised to $\beta$-actin (Hs_ACTB_1_SG QuantiTect Primer Assay [NM_001101] Qiagen, Manchester, UK).

\section{Sanger sequencing}

Sanger sequencing in forward and reverse orientations was performed by Source Biosciences (Oxford) using the sequencing primer: 5'-GTT TCC ATT CCA CCA ACA CC-3'.

\section{Statistical analysis}

Association data for genotyped and imputed SNPs were obtained on the subset of AS cases of white British ancestry and 
white British controls from the recent ImmunoChip GWAS. To evaluate the presence of independent effects on genetic susceptibility at the RUNX3 locus, we performed conditional analysis on 4230 AS cases and 9700 matched controls, as previously described. ${ }^{1}$ Association analysis was performed using the logistic regression function in PLINK (V.1.90), ${ }^{27}$ accounting for population structure with $10 \mathrm{PCs}$, and conditioning on the RUNX3 SNP $r s 4648889$.

One-way analysis of variance (ANOVA) and two-tailed Student's $t$ test were used to determine statistical significance using the GraphPad Prism software (V.5.03) package.

\section{RESULTS}

\section{Conditional analysis identifies rs4648889 as a candidate causal variant}

The previous AS ImmunoChip study ${ }^{1}$ identified $r s 6600247$ as the lead SNP at the RUNX3 locus $\left(1.3 \times 10^{-14}\right)$, with $r s 4648889$ as the next most strongly associated SNP $\left(p=7.6 \times 10^{-14}\right)$. We performed conditional analysis and found no evidence of independent effects in disease susceptibility between these two SNPs, reflecting the strong LD between them (table 1). After conditioning on SNP rs4648889, only two of the other 22 RUNX3 SNPs previously shown to be strongly associated with AS at $\mathrm{p} \leq 10^{-11}$ retained positive $(\mathrm{p}<0.02)$ associationrs4265380 $\left(1.7 \times 10^{-7}\right)$ and $r s 7529070 \quad\left(\mathrm{p}=1.2 \times 10^{-7}\right)$. Conditioning on these two SNPs independently, the strong association with the SNP $r s 4648889$ was retained $\left(\mathrm{p} \leq 3.0 \times 10^{-6}\right.$ for both SNP), thereby establishing the primacy of this association with AS in this region.

\section{Identification of a regulatory region upstream of RUNX3 containing rs4648889}

We first identified a putative regulatory element $\sim 2 \mathrm{~kb}$ upstream of the RUNX3 distal promoter in the region of $r s 4648889$ (figure 1A). This was based on published DNase I hypersensitivity sites (DHS) in seven cell types, ChIP-seq peaks for TF binding and the presence of both $\mathrm{H} 3 \mathrm{~K} 4 \mathrm{Me} 1$ and $\mathrm{H} 3 \mathrm{~K} 27 \mathrm{Ac}$ histone modifications (figure 1A). ${ }^{20} 28$ This regulatory element also contains a DHS site in CD8 + T cells (http://www.epigenomebrowser.org) and is predicted to bind several TFs. The region around rs4648889 is more likely to be functionally relevant than rs6600247, the lead SNP in the previous ImmunoChip study, considering the paucity of TF binding near the latter (figure 1B).
We therefore hypothesised that this region is an active enhancerlike element $5^{\prime}$ of the RUNX3 promoter (figure 1).

\section{Differential binding of nuclear extract including IRF4 at rs4648889}

Analysis of ChIP-seq data from the ENCODE project revealed binding of several TFs to the region surrounding rs4648889 (figure 1). We investigated the effect of the $r s 4648889$ dimorphism on TF binding to a $50 \mathrm{bp}$ DNA fragment using EMSA. Addition of nuclear extract from Jurkat cells (leukaemia T-cell line) to this DNA probe created a major protein-DNA complex (i) with a visibly weaker band for the AS-risk allele ' $A$ ' ( $\sim 5.4$-fold \pm 1.2 SEM less than ' $G$ ') than the AS-protective ' $G$ ' allele (figure 2A). Addition of CD8 + T-cell nuclear extract also generated a weaker band for a protein-DNA complex (ii) with the risk 'A' allele ( 3.1-fold \pm 1.4 SEM less than ' $G$ ') compared with the ' $G$ ' allele (figure $2 \mathrm{~B}$ ). In these experiments Jurkat and $\mathrm{CD} 8+$ T-cell nuclear extract binding to the ' $G$ ' allele was successfully competed by 200 -fold and 100-fold excess of unlabelled ' $G$ ', but not 'A' probe and vice versa (figure $2 \mathrm{~A}$ and see online supplementary figure S1A). The strong binding of both Jurkat and CD8+ T-cell nuclear lysates to the AS-protective ' $G$ ' allele of $r s 4648889$ was greatly reduced by adding IRF4 antibody. In contrast, this had little discernible influence on the already very weak binding of either of these nuclear lysates to the AS-risk 'A' allele (figure 2A, B).

This suggests that TFs, including IRF4 in the resulting complex, exhibit allele-specific binding to the region around rs4648889.

\section{rs4648889 alters IRF4 binding and H3K4Me1 histone methylation}

We used ChIP and allele-specific qPCR to assess DNA binding by IRF4 in rs4648889 heterozygous CD8 + T cells freshly isolated from three patients with AS. Three independent experiments showed IRF4 was preferentially recruited (threefold increase \pm 1.0 SEM, $p=0.058$ ) to the AS-protective ' $G$ ' allele compared with the AS-risk 'A' allele (figure 3A).

ChIP-seq data from ENCODE identified the $2 \mathrm{~kb}$ region upstream of the RUNX3 promoter as an enhancer region with increased histone $\mathrm{H} 3 \mathrm{~K} 4 \mathrm{Me} 1$ in seven different cell lines. We therefore conducted ChIP qPCR for H3K4Me1 on CD8 + T

\begin{tabular}{|c|c|c|c|c|c|c|c|c|}
\hline \multirow[t]{2}{*}{1} & rs6600247 & 25177701 & $\mathrm{C} / \mathrm{T}$ & rs4648889 & 0.9 & 1.01 & $0.54 / 0.51$ & $0.90 / 0.97$ \\
\hline & & & & rs7529070 & $4.9 \times 10^{-5}$ & 1.67 & & $0.97 / 1$ \\
\hline \multirow[t]{2}{*}{1} & rs 4648889 & 25166416 & $\mathrm{~A} / \mathrm{G}$ & rs6600247 & 0.2 & 0.84 & $0.54 / 0.50$ & $0.90 / 0.97$ \\
\hline & & & & rs 4265380 & $2.1 \times 10^{-6}$ & 3.96 & & $0.94 / 1$ \\
\hline \multirow[t]{3}{*}{1} & $r s 4265380$ & 25165943 & $\mathrm{~T} / \mathrm{C}$ & rs6600247 & $1.0 \times 10^{-6}$ & 0.53 & $0.55 / 0.50$ & $0.97 / 1$ \\
\hline & & & & $r s 4648889$ & $1.7 \times 10^{-7}$ & 0.22 & & $0.94 / 1$ \\
\hline & & & & rs7529070 & 0.6 & 1.14 & & $1 / 1$ \\
\hline \multirow[t]{2}{*}{1} & rs7529070 & 25168167 & $A / G$ & rs6600247 & $2.1 \times 10^{-7}$ & 0.52 & $0.53 / 0.50$ & $0.97 / 1$ \\
\hline & & & & rs4648889 & $1.2 \times 10^{-7}$ & 0.30 & & $0.94 / 1$ \\
\hline
\end{tabular}

${ }^{*}$ National Center for Biotechnology Information (NCBI) Build 36 human genome coordinates.

Chr., chromosome; LD, linkage disequilibrium; RAF, risk allele frequency; SNP, single nucleotide polymorphism. 
Figure 1 Epigenetic and transcriptional landscape of the $2 \mathrm{~kb}$ putative regulatory region containing rs4648889 upstream of the RUNX3 promoter. (A) Representation of RUNX3 promoter and putative regulatory element (PRE) location (a). From ENCODE data: RUNX3 gene location and promoter (b). Histone modification chromatin immunoprecipitation (ChIP)-seq data including H3K4me1 and H3K27Ac (c). DNase I hypersensitivity GM12878 lymphoblastoid cell line (d), predicted chromatin state (e) and condensed transcription factor (TF) ChIP-seq (f) data from various cell lines are shown. (B) rs4648889 site shows specific TF binding compared with rs6600247. ENCODE TF ChIP-seq data from GM12878 lymphoblastoid cell line show differential TF binding between rs4648889 and rs6600247 for RUNX3, IRF4, IKZF1, NFATC1 and SPIB. Blue and light violet dots represent genotyped and imputed single nucleotide polymorphisms variants and their location; light brown rectangle shows the RUNX3 promoter.
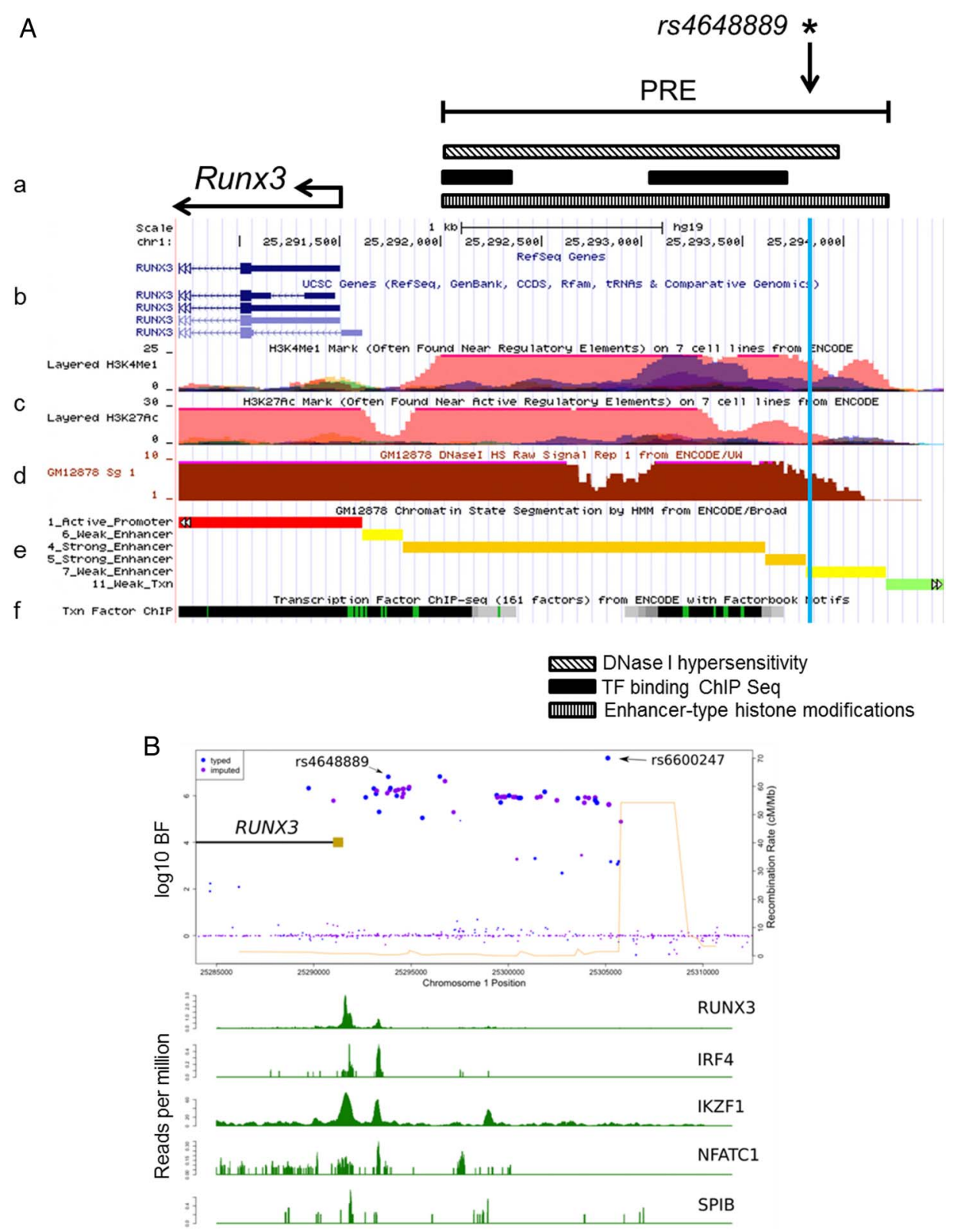

cells. The AS-protective ' $G$ ' allele had $>2.5$-fold relative enrichment $(p=0.03)$ compared with the AS-risk 'A' allele (figure 3B).

Sanger sequencing of one rs4648889-heterozygous AS case showed that the ' $G$ ' allele was relatively enriched in chromatin fragments immunoprecipitated with antibodies for H3K4Me1 and IRF4 ChIP compared with the reference input (see online supplementary figure S2).

\section{The AS-risk 'A' allele at rs4648889 shows decreased reporter gene activity}

Reporter activity of the region around $r s 4648889$ was also evaluated in vitro by luciferase reporter assay. The $250 \mathrm{bp}$ region containing rs 4648889 showed increased luciferase activity compared with the minimal promoter $(\operatorname{minP}=1)$ but the presence of the AS-risk allele 'A' reduced the enhancer activity from 9.1-fold to 4.3 -fold $(\mathrm{p} \leq 0.01)$ in HEK293T cells (human embryonic kidney cell line) and from 4.0 -fold to 1.9 -fold $(\mathrm{p} \leq 0.01)$ in Jurkat cells (figure 4A, B). The same result was observed in Jurkat cells stimulated with phorbol myristate acetate (PMA)/ phytohaemaglutinin (PHA) for $24 \mathrm{~h}$ (figure 4C). These findings support the view that the lower enhancer activity at the AS-risk 'A' allele is due to decreased H3K4Me1 occupancy.

\section{The homozygous 'AA' genotype at rs4648889 is associated with reduced $R U N X 3$ mRNA levels}

Analysis of RUNX3 expression (figure 5) in CD8 + T cells isolated from patients with AS having different rs4648889 genotypes (6 GG, $7 \mathrm{AG}$ and $6 \mathrm{AA}$ ) showed that RUNX3 mRNA levels, normalised against $\beta$-actin, were significantly lower in subjects with the AA than the GG genotype $(1.7 \pm 0.9$ vs 4.2 \pm 2.2 SEM, ANOVA $\mathrm{p} \leq 0.05)$. No correlation was observed between RUNX3 expression and disease activity as defined either by BASDAI or CRP.

\section{DISCUSSION}

We have shown here that $r s 4648889$ is probably the primary AS-associated SNP at the RUNX3 locus and that this association can probably be best explained through its effects on gene expression. The $r s 4648889$ AS-risk allele 'A' exhibits relatively low luciferase reporter activity compared with the 

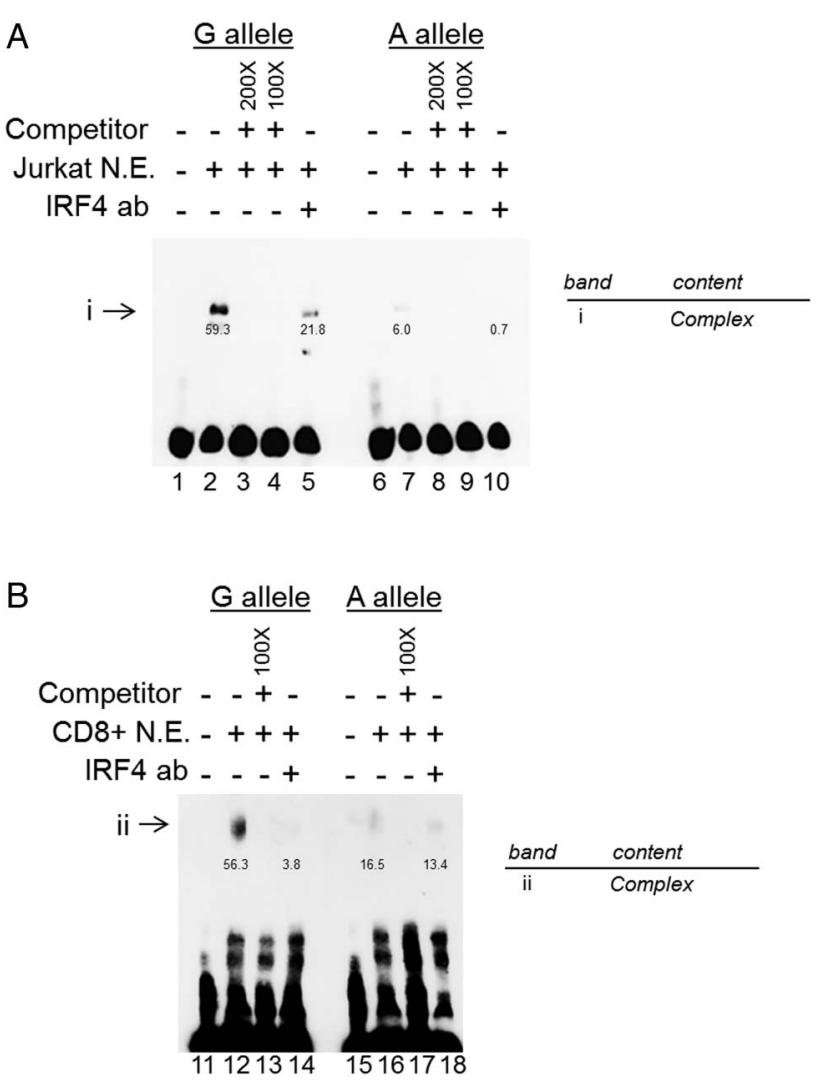

Figure 2 rs4648889 segment region alters protein-DNA complex formation with evidence of IRF4 involvement. Chemiluminescent electrophoretic mobility gel shift assay (EMSA) (A) showing complex formations (i) after addition of Jurkat nuclear extract (lanes 2 and 7), competition with 200 -fold and 100 -fold excess of unlabelled probes (lanes 3, 4 and 8,9) and IRF4 involvement after addition of IRF4 antibody (lanes 5 and 10). IRF4 antibody addition leads to inhibition of the complex (i) (lanes 5 and 10). (B) EMSA showing protein-DNA complex formation (ii) after addition of CD8+ nuclear extract (lanes 12 and 16). IRF4 antibody was used to compete with the labelled oligonucleotides for binding of the nuclear extract (lanes 14-18). Numbers below the bands indicate pixel density. N.E., nuclear extract.

AS-protective 'G' allele, less RUNX3 mRNA levels and less enrichment for $\mathrm{H} 3 \mathrm{~K} 4 \mathrm{Me} 1$ histone modification of a type associated with active enhancer elements. ${ }^{7}$ The AS-risk allele ' $A$ ' is also reproducibly associated with reduced formation of protein-DNA complexes that contain IRF4. The TF IRF4 is involved in regulating the number and function of CD8 $+\mathrm{T}$ cells, ${ }^{29-32}$ influencing their differentiation, expansion ${ }^{30} 33$ and metabolism. ${ }^{31}$ Our results are consistent with previous reports that selective IRF4 binding to the distal promoter exerts an inhibitory effect on RUNX3 expression and consequently on $\mathrm{CD} 8+$ T-cell development and function. ${ }^{32}$ Previous studies have indicated that the total number of peripheral blood CD8+ $\mathrm{T}$ cells is significantly decreased in AS cases, and that this is strongly correlated with the RUNX3 genotype. ${ }^{1}{ }^{2}$ We believe that this may be mediated through differential allelic binding of TF complexes (including IRF4) to this RUNX3 enhancer. RUNX3 plays a key role in haematopoiesis and enhances CD8 T-cell development/activation and has an inhibitory effect on CD4+ T cells. ${ }^{9}{ }^{10}$ RUNX3 affects NK cell development, ${ }^{10}$ mediates transforming growth factor- $\beta$ responses in the activation of dendritic cells during inflammation ${ }^{34}$ and, together with T-bet, regulates interferon- $\gamma$ and interleukin 4 expression in T-helper (Th) 1 cells. $^{35}$
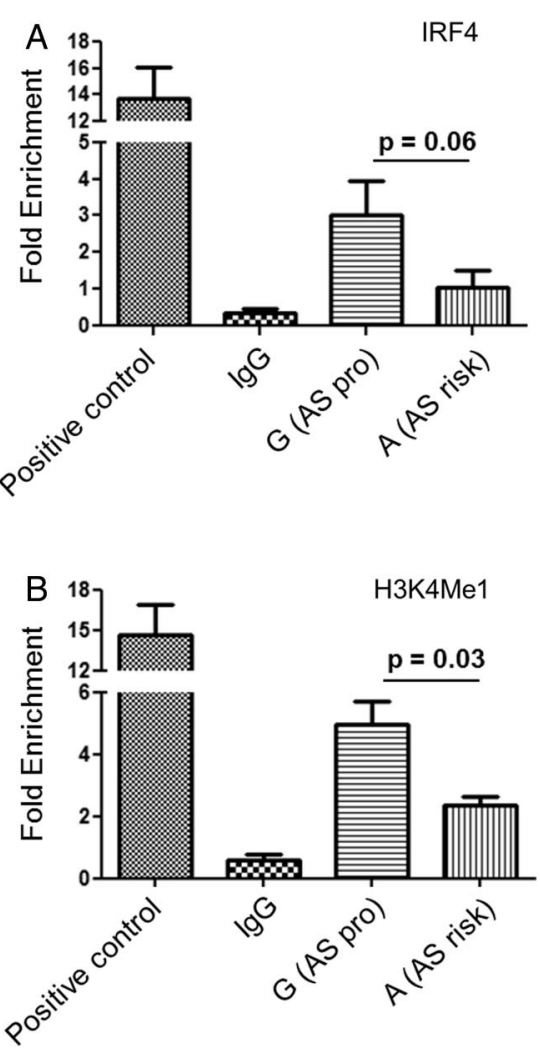

Figure 3 Ex vivo allele-specific binding of IRF4 and H3K4Me1 methylation at the $r \$ 648889$ site. Allele-specific IRF4 (A) and H3K4Me1 (B) chromatin immunoprecipitation (ChIP) quantitative PCR assessed at the rs4648889 locus in heterozygous CD8+ T cells isolated from three patients with ankylosing spondylitis (AS). The PCR reactions for both the ' $G$ ' and ' $A$ ' alleles were done in triplicate in each of the three cases. The relative enrichment is expressed as mean $\pm S E M$ ( $p<0.05$, Student's t test). Enhancer sequence from IL10 was used as positive control.

The striking $H L A-B * 27$ association with AS has long tempted speculation that aberrant immune responses to microbial infection, perhaps mediated through HLA-B27-restricted CD8 + cytotoxic $\mathrm{T}$ cells, could be involved. ${ }^{36} 37$ Such ideas have been strengthened by the observation that $H L A-B * 27$-transgenic rats develop SpA unless reared in germ-free conditions. ${ }^{38}$ However, $H L A-B * 27$ transgenic rats still develop $\mathrm{SpA}$ in the absence of CD8 + T cells. ${ }^{39}$ Our results would be consistent with the hypothesis that reduced CD8 + T-cell numbers and/or function play a role in the pathogenesis of AS. More than 40 genetic influences have been identified in AS, several of which (HLA-B*27, RUNX3, EOMES, TBX21, ZMIZ1, IL7 and IL7R) potentially affect lymphocyte biology. ${ }^{1}$ Currently, the full complexity of immune cell involvement in AS is incompletely understood. Here we have demonstrated potential epigenetic regulatory effects at the RUNX3 locus in CD8 $+\mathrm{T}$ cells. We have not yet extended these studies to other cell types, such as Th1, NK, dendritic or other immune cells that could also be involved in AS. ${ }^{40}$ For example, reduced RUNX3 expression could enhance CD4+ T-cell activity, which would be consistent with models of AS invoking a pathological role for CD4+ T cells. ${ }^{41}{ }^{42}$ In particular, CD4+ Th17 cells have been implicated in AS and appear to be present in increased numbers in the peripheral blood of patients with pre-radiographical axial $\mathrm{SpA}^{43}$ and reactive arthritis. ${ }^{44}$

It is likely that most genetic associations in AS reflect relatively subtle changes in gene expression. The differences in 

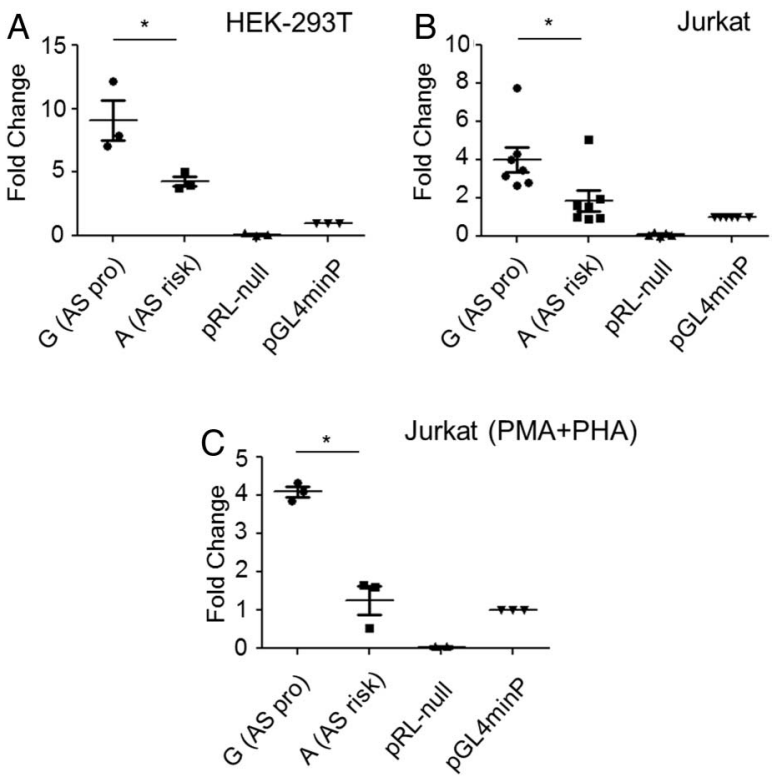

Figure 4 rs4648889 site shows enhancer activity in vitro. The transcriptional activity of $r s 4648889$ compared with minimal promoter $(\operatorname{minP})$ was measured by luciferase assays on (A) HEK293T $(n=3),(B)$ Jurkat cells $(n=7)$ and $(C)$ Jurkat cells stimulated with PMA/PHA for $24 \mathrm{~h}(\mathrm{n}=3)$. The values of relative luciferase activity are expressed as mean $\pm S E M$. The relative luciferase activity of the ankylosing spondylitis (AS)-risk 'A' allele was significantly reduced compared with the AS-protective ' $G$ ' allele ( ${ }^{*} p<0.01$, Student's t test).

DNA-protein complexes revealed by EMSA experiments between the AS-risk ' $\mathrm{A}$ ' and AS-protective ' $G$ ' allele require further investigation to define the full diversity of TFs involved in these complexes and also the network of genes that might ultimately be regulated by the rs4648889 polymorphism. It is

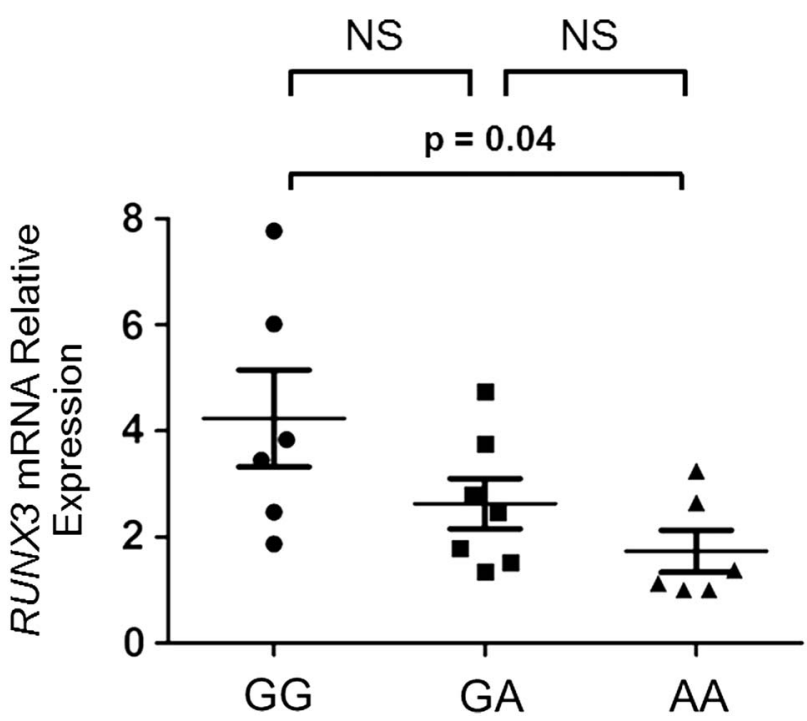

Figure 5 Allele-specific effects of $r$ 44648889 on RUNX3 expression. Relative amount of RUNX3 mRNA transcript in primary CD8+ T cells from 19 patients with ankylosing spondylitis having different genotypes was measured by quantitative PCR using the comparative cycle threshold method. The $p$ values were determined using analysis of variance. There was a significant difference $(p=0.04)$ between the $G G$ and the AA homozygotes. The differences between the GA heterozygotes and the GG or AA homozygotes were not significant (NS). well known that eukaryotic transcription may be controlled by regulatory elements that are distant from their target genes; such control can be exerted (sometimes on multiple genes) through the formation of chromatin loops. ${ }^{45} 46$ We have not formally excluded the possibility that this RUNX3 regulatory region could influence other more distant genes relevant to AS. Establishing the full extent of the genes (and their regulation) in these pathways and their relationship to the pathogenesis of AS represents a considerable challenge. In future we will investigate TF binding and chromatin accessibility across the whole genome to explore the composition of these AS gene regulatory networks. $^{47}$

This work illustrates the first steps towards a more complete mechanistic understanding of just one of the many genetic associations of AS. These and related techniques have broad applications to the investigation of other similar regulatory polymorphisms that probably account for much of the genetic risk in AS. A deeper knowledge of the factors involved in these processes is likely to lead of the discovery of new therapeutic targets.

Acknowledgements The authors wish to thank all the study participants who generously donated their DNA to this study.

Contributors MV, ARR, CJC, AC, JCK, PB and BPW conceived and designed the experiments. MV, ARR and AC performed the experiments. MV, ARR, CJC, AC, PB and $B P W$ analysed the data. MV, ARR, CJC, AC, PB, JCK and BPW wrote the manuscript.

Funding MV was funded by NIHR Oxford Comprehensive Biomedical Research Centre (immunity and inflammation theme A93081) and ARR by Arthritis Research UK (Grant 20402). Additional funding was provided by Arthritis Research UK (Grants 18797, 19536 and 20796), the NIHR Thames Valley collaborative research network and National Ankylosing Spondylitis Society (UK).

Competing interests None declared.

Patient consent Obtained.

Ethics approval Central Oxford Research Ethics Committee COREC 06/Q1606/139 and OXREC B 07/Q1605/35).

Provenance and peer review Not commissioned; externally peer reviewed.

Open Access This is an Open Access article distributed in accordance with the terms of the Creative Commons Attribution (CC BY 4.0) license, which permits others to distribute, remix, adapt and build upon this work, for commercial use, provided the original work is properly cited. See: http://creativecommons.org/ licenses/by/4.0/

\section{REFERENCES}

1 Cortes A, Hadler J, Pointon JP, et al., International Genetics of Ankylosing Spondylitis Consortium (IGAS). Identification of multiple risk variants for ankylosing spondylitis through high-density genotyping of immune-related loci. Nat Genet 2013:45:730-8.

2 Evans DM, Spencer CC, Pointon JJ, et al. Interaction between ERAP1 and HLA-B27 in ankylosing spondylitis implicates peptide handling in the mechanism for HLA-B27 in disease susceptibility. Nat Genet 2011;43:761-7.

3 Karaderi T, Harvey D, Farrar C, et al. Association between the interleukin 23 receptor and ankylosing spondylitis is confirmed by a new UK case-control study and meta-analysis of published series. Rheumatology (Oxford) 2009;48:386-9.

4 Harvey D, Pointon JJ, Evans DM, et al. Investigating the genetic association between ERAP1 and ankylosing spondylitis. Hum Mol Genet 2009;18:4204-12.

5 Hindorff LA, Sethupathy P, Junkins HA, et al. Potential etiologic and functional implications of genome-wide association loci for human diseases and traits. Proc Natl Acad Sci USA 2009;106:9362-7.

6 Apel M, Uebe S, Bowes J, et al. Variants in RUNX3 contribute to susceptibility to psoriatic arthritis, exhibiting further common ground with ankylosing spondylitis. Arthritis Rheum 2013:65:1224-31.

7 Schaub MA, Boyle AP, Kundaje A, et al. Linking disease associations with regulatory information in the human genome. Genome Res 2012:22:1748-59.

8 Cohen MM Jr. Perspectives on RUNX genes: an update. Am J Med Genet 2009; 149:2629-46.

9 Lotem J, Levanon D, Negreanu V, et al. Runx3 at the interface of immunity, inflammation and cancer. Biochim Biophys Acta 2015;1855:131-43.

10 Levanon D, Negreanu V, Lotem J, et al. Transcription factor Runx3 regulates interleukin-15-dependent natural killer cell activation. Mol Cell Biol 2014;34:1158-69. 
11 Sugai $M$, Aoki K, Osato $M$, et al. Runx3 is required for full activation of regulatory $T$ cells to prevent colitis-associated tumor formation. J Immunol 2011;186: 6515-20.

12 Taniuchi I, Osato M, Egawa $T$, et al. Differential requirements for Runx proteins in CD4 repression and epigenetic silencing during T lymphocyte development. Cell 2002:111:621-33.

13 Cruz-Guilloty F, Pipkin ME, Djuretic IM, et al. Runx3 and T-box proteins cooperate to establish the transcriptional program of effector CTLs. J Exp Med 2009;206:51-9.

14 Rini D, Calabi F. Identification and comparative analysis of a second RUNX3 promoter. Gene 2001:273:13-22.

15 Bangsow C, Rubins N, Glusman G, et al. The RUNX3 gene sequence, structure and regulated expression. Gene 2001;279:221-32.

16 Han H, Cortez CC, Yang $\mathrm{X}$, et al. DNA methylation directly silences genes with non- $\mathrm{CpG}$ island promoters and establishes a nucleosome occupied promoter. Hum Mol Genet 2011;20:4299-310.

17 Paluszczak J, Sarbak J, Kostrzewska-Poczekaj M, et al. The negative regulators of Wnt pathway-DACH1, DKK1, and WIF1 are methylated in oral and oropharyngeal cancer and WIF1 methylation predicts shorter survival. Tumour Biol 2015;36:2855-61.

18 Estécio MR, Maddipoti S, Bueso-Ramos C, et al. RUNX3 promoter hypermethylation is frequent in leukaemia cell lines and associated with acute myeloid leukaemia inv (16) subtype. Br J Haematol 2015;169:344-51.

19 Guo C, Ren F, Wang D, et al. RUNX3 is inactivated by promoter hypermethylation in malignant transformation of ovarian endometriosis. Oncol Rep 2014;32:2580-8.

20 ENCODE Project Consortium. A user's guide to the encyclopedia of DNA elements (ENCODE). PLOS Biol 2011;9:e1001046.

21 Delaneau 0, Zagury JF, Marchini J. Improved whole chromosome phasing for disease and population genetic studies. Nat Methods 2013;10:5-6.

22 Abecasis GR, Auton A, Brooks LD, et al., 1000 Genomes Project Consortium. An integrated map of genetic variation from 1,092 human genomes. Nature 2012:491:56-65.

23 Howie B, Fuchsberger C, Stephens M, et al. Fast and accurate genotype imputation in genome-wide association studies through pre-phasing. Nat Genet 2012;44:955-9.

24 Howie BN, Donnelly P, Marchini J. A flexible and accurate genotype imputation method for the next generation of genome-wide association studies. PLoS Genet 2009:5:e1000529.

25 Van der Linden S, Valkenburg HA, Cats A. Evaluation of diagnostic criteria for ankylosing spondylitis. A proposal for modification of the New York criteria. Arthritis Rheum 1984;27:361-8.

26 Garrett S, Jenkinson T, Kennedy LG, et al. A new approach to defining disease status in ankylosing spondylitis: the Bath Ankylosing Spondylitis Disease Activity Index. J Rheumatol 1994;21:2286-91.

27 Purcell S, Neale B, Todd-Brown K, et al. PLINK: a tool set for whole-genome association and population-based linkage analyses. Am J Hum Genet 2007:81:559-75

28 ENCODE Project Consortium. An integrated encyclopedia of DNA elements in the human genome. Nature 2012;489:57-74.
29 Lotem J, Levanon D, Negreanu V, et al. Runx3-mediated transcriptional program in cytotoxic lymphocytes. PLOS ONE 2013;8:e80467.

30 Yao S, Buzo BF, Pham D, et al. Interferon regulatory factor 4 sustains CD8(+) T cell expansion and effector differentiation. Immunity 2013;39:833-45.

31 Man K, Miasari M, Shi W, et al. The transcription factor IRF4 is essential for TCR affinity-mediated metabolic programming and clonal expansion of $\mathrm{T}$ cells. Nat Immunol 2013;14:1155-65.

32 Cao $Y$, Li H, Sun $Y$, et al. Interferon regulatory factor 4 regulates thymocyte differentiation by repressing Runx3 expression. Eur J Immunol 2010;40:3198-209.

33 Nayar R, Schutten E, Bautista B, et al. Graded levels of IRF4 regulate CD8+T cell differentiation and expansion, but not attrition, in response to acute virus infection. J Immunol 2014;192:5881-93.

34 Fainaru $\mathrm{O}$, Woolf $\mathrm{E}$, Lotem J, et al. Runx3 regulates mouse TGF-beta-mediated dendritic cell function and its absence results in airway inflammation. EMBO J 2004;23:969-79.

35 Collins PL, Chang S, Henderson M, et al. Distal regions of the human IFNG locus direct cell type-specific expression. J Immunol 2010;185:1492-501.

36 Fiorillo MT, Maragno M, Butler R, et al. CD8(+) T-cell autoreactivity to an HLA-B27-restricted self-epitope correlates with ankylosing spondylitis. J Clin Invest 2000;106:47-53.

37 Schirmer M, Goldberger C, Würzner R, et al. Circulating cytotoxic CD8(+) CD28(-) T cells in ankylosing spondylitis. Arthritis Res 2002;4:71-6.

38 Taurog JD, Richardson JA, Croft JT, et al. The germfree state prevents development of gut and joint inflammatory disease in HLA-B27 transgenic rats. J Exp Med 1994; 180:2359-64.

39 Taurog JD, Dorris ML, Satumtira N, et al. Spondylarthritis in HLA-B27/human beta2-microglobulin-transgenic rats is not prevented by lack of CD8. Arthritis Rheum 2009:60:1977-84

40 Chan AT, Kollnberger SD, Wedderburn LR, et al. Expansion and enhanced survival of natural killer cells expressing the killer immunoglobulin-like receptor KIR3DL2 in spondylarthritis. Arthritis Rheum 2005;52:3586-95.

41 Overgaard NH, Jung J, Steptoe RJ, et al. CD4+/CD8+ double-positive T cells: more than just a developmental stage? J Leukoc Biol 2015;97:31-8.

42 Boyle LH, Goodall JC, Gaston JS. The recognition of abnormal forms of HLA-B27 by CD4+ T cells. Curr Mol Med 2004:4:51-8.

43 Jansen DT, Hameetman M, van Bergen J, et al. IL-17-producing CD4+ T cells are increased in early, active axial spondyloarthritis including patients without imaging abnormalities. Rheumatology (Oxford) 2015;54:728-35.

44 Shen H, Goodall JC, Gaston JS. Frequency and phenotype of Thelper 17 cells in peripheral blood and synovial fluid of patients with reactive arthritis. J Rheumatol 2010;37:2096-9.

45 Ghoussaini M, Edwards SL, Michailidou K, et al. Evidence that breast cancer risk at the 2 q35 locus is mediated through IGFBP5 regulation. Nat Commun 2014;4:4999.

46 Huang Q, Whitington T, Gao P, et al. A prostate cancer susceptibility allele at $6 \mathrm{q} 22$ increases RFX6 expression by modulating HOXB13 chromatin binding. Nat Genet 2014:46:126-35

47 Ciofani M, Madar A, Galan C, et al. A validated regulatory network for Th17 cell specification. Cell 2012;151:289-303. 